\title{
Die Ökonomik der Weihnachtsgeschenke
}

Wer kennt sie nicht, die Freude, die einen überkommt, wenn man am Weihnachtsabend das Geschenk, das man von der Schwester oder dem Bruder erhalten hat, auspackt und feststellt, dass es sich wieder um einen grauen Schal mit einem aufregenden hellgrauen Streifen handelt, den man dann ordentlich gefaltet zu den anderen Schals der Vorjahre legen kann. Man selbst hat vermutlich auch schon etliche Male ähnliche Gefühle ausgelöst durch das Verschenken nicht passender Kleidungsstücke, nichtssagender Gebrauchsgegenstände oder von Deko-Artikeln, die niemand braucht und die nach Weihnachten irgendwo im Weg stehen. Nein, es kommt ganz sicher häufig vor, dass man das Geld, das für ein Geschenk ausgegeben wird, besser dem Beschenkten in bar ausgezahlt hätte, auf dass er oder sie sich selbst etwas kauft, was dann wirklich nützlich ist. Zu dieser weihnachtlichen Allerweltserfahrung gibt es eine ökonomische Theorie. Joel Waldfogel hat sie 1993 in einem Artikel in der American Economic Review in die Welt gesetzt, sie beschreibt den „Deadweight Loss of Christmas“, also den Effizienzverlust, der dadurch entsteht, dass man Geschenke „in kind“ macht, und dabei häufig daneben liegt, anstatt Geschenke „in cash“ zu machen, die dem Beschenkten erlauben, das Geschenk perfekt an seine Präferenzen anzupassen.

Waldfogel hat mit seinem Artikel eine akademische Diskussion angestoßen, die zehn Jahre anhielt und einige Artikel in der American Economic Review produziert hat. In dieser Diskussion ging es vor allem um die Frage, wie man methodisch vorgehen soll, um den Effizienzverlust durch Weihnachtsgeschenke abzuschätzen. Waldfogel selbst kommt immerhin auf Größenordnungen von etwa 4 bis $13 \mathrm{Mrd}$. US-\$. Das entspricht einem Zehntel bis einem Drittel des Effizienzverlustes, der damals in den USA durch Besteuerung ausgelöst wurde. Da lohnt es sich doch einmal genauer hinzusehen. Hier soll es aber um eine andere Frage gehen, die sich ebenfalls aufdrängt: Kann man sich vorstellen, dass in einer freiheitlichen Gesellschaft, die ihre Ökonomie weitgehend über Märkte reguliert, eine Institution Bestand haben kann, die zu solch enormen Effizienzverlusten führt? Reagieren Märkte nicht auf solche Ineffizienzen? Und ist die Ineffizienz wirklich so hoch? Beginnen wir mit der Reaktion der Märkte.

Ökonomen glauben daran, dass kein Geld auf der Straße liegen bleibt. Wenn Effizienzgewinne möglich sind, dann werden sie auch früher oder später realisiert. Zwei Marktreaktionen, die versuchen, die Ineffizienz beim Schenken zu reduzieren, seien beispielhaft genannt. Die erste besteht darin, Wertgutscheine zu verkaufen, anstatt Waren. Für den Beschenkten hat das den Vorteil, dass er sich aus dem Sortiment selbst etwas aussuchen kann und das reduziert natürlich den Effizienzverlust. Für den Verkäufer entsteht auch ein Vorteil, denn ein kleiner Teil der Gutscheine wird nicht eingelöst und in vielen Fällen legen die mit dem Gutschein Beschenkten „noch etwas drauf" und kaufen das etwas höherwertige Handy oder den besseren Pullover. Eine zweite Reaktion richtet sich beispielsweise an die Männer, die alljährlich vor dem Problem stehen, ein möglichst passendes Geschenk für die Partnerin zu finden. Clevere Schmuckdesigner haben dafür Sammlerkollektionen erfunden, die es erlauben, jedes Jahr ein neues Geschenk , in kind“ zu machen, von dem Mann sehr genau weiß, dass es den Präferenzen der Beschenkten entsprechen wird, weil es die Schmucksteinsammlung ergänzt, von der bekannt ist, dass sie gefällt.

Trotz dieser Marktreaktionen bleiben noch eine Menge Geschenke über, die nicht aus Gutscheinen oder Sammlungsergänzungen bestehen. Es muss dafür einen Grund

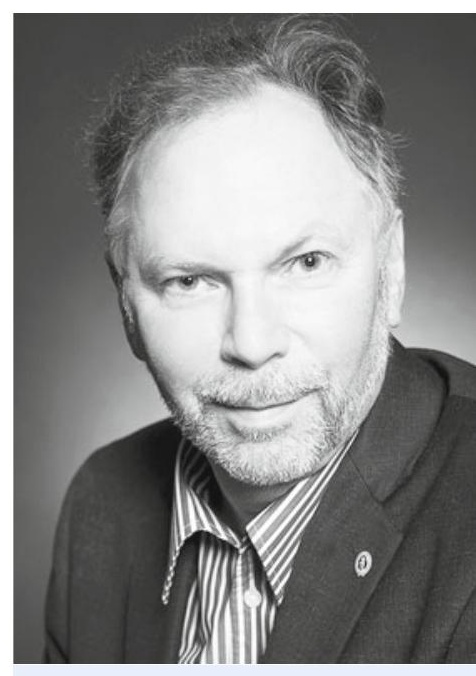

Joachim Weimann lehrt Wirtschaftspolitik an der Otto-von-Guericke-Universität in Magdeburg. 
geben. Zunächst ist es hilfreich zu beachten, dass Geschenke häufig in reziproken Relationen gemacht werden, d.h. man beschenkt sich gegenseitig. Weiterhin ist es sinnvoll, zwischen symmetrischen und asymmetrischen Geschenkrelationen zu unterscheiden. Einfach zu erklären sind asymmetrische Geschenke „in kind“. Eltern schenken ihren noch jungen Kindern etwas, weil sie damit einen pädagogischen Auftrag erfüllen. Erstens signalisieren sie ihren Kindern damit Zuneigung und zweitens betätigen sie sich als (hoffentlich) pädagogisch verantwortungsvolle Sachwalter der Interessen der Kinder. Wohl nur sehr wenige Eltern kommen auf die Idee einem fünfjährigen Kind Geld in die Hand zu drücken, damit er oder sie sich davon etwas zu Weihnachten kaufe.

Schwieriger zu erklären sind symmetrische Geschenkrelationen beispielsweise zwischen Ehepartnern. Folgt man Waldfogel, so bestünde eine effiziente Lösung darin, unter dem Weihnachtsbaum hübsch verpackte 100-Euro-Scheine auszutauschen, oder - um die Verpackungskosten zu sparen - ganz auf Geschenke zu verzichten. Wir beobachten aber, dass es dazu vielfach nicht kommt. Das spricht dafür, dass der Austausch von Geschenken zu einem Effizienzgewinn führt. In der verhaltensökonomischen Laborforschung gibt es dafür ein Spiel. Im „Mutual Gift Giving Game“ bekommen zwei Versuchspersonen jeweils 10 Euro ausgehändigt. Sie können davon beliebig viel an den anderen Spieler abgeben (verschenken). Jeder Euro, der abgegeben wird, wird vom Experimentator verdoppelt. Auf diese Weise entsteht ein Effizienzgewinn durch Schenken und die kollektiv rationale Lösung besteht darin, dass beide Spieler dem andern jeweils ihre 10 Euro schenken. Damit stellt sich die Frage, worin der analoge Effizienzgewinn beim Austausch von Weihnachtsgeschenken bestehen könnte. Eine Hypothese könnte sein, dass in reziproken Beziehungen auch der Schenkende einen Vorteil aus dem Geschenk hat. Beispielsweise kann er mit einem Geschenk signalisieren, dass er sich intensiv Gedanken darüber gemacht hat, was für den Partner vorteilhaft sein könnte. Er kann mit einem Geschenk Wertschätzung und Anerkennung verbinden, die wiederum mit Anerkennung und Dank vergolten werden. Auf diese Weise bieten reziproke Beziehungen Möglichkeiten, durch gegenseitige Geschenke einen Effizienzgewinn zu realisieren.

Ein weiterer Punkt kommt hinzu. Aus der experimentellen Forschung wissen wir, dass es für Menschen wichtig ist, bestimmte Erfahrungen unmittelbar zu machen und nicht hypothetisch zu erleben. Verschenkt man einen Gutschein oder Geld, erlebt man nicht, wie der Beschenkte sich über die Sache freut, die er letztlich durch das Geschenk bekommt. Man weiß nur „hypothetisch“ dass der Beschenkte sich freuen wird, wenn er das Geschenk letztendlich in Händen hält. Brosig et al. haben 2003 beispielsweise festgestellt, dass es so etwas wie einen "hot versus cold effect" gibt und es einen Unterschied macht, ob man unmittelbar erlebt, was jemand tut oder nur hypothetisch. Imas zeigt 2014 in seinem Aufsatz „The realization effect: risk-taking after realized versus paper losses“, dass die Reaktion auf Verluste davon abhängt, ob man Geld unmittelbar verliert, oder nur einen „Buchverlust“ erleidet. In letzterem Fall besteht die Reaktion darin, verstärkt in riskante Anlagen zu investieren, in ersterem werden die Akteure risikoscheuer. Es kommt also sehr darauf an, Dinge unmittelbar und sensitiv zu erfahren. Warum sollte dies bei Geschenken anders sein? Sass und Weimann kommen 2014 jedenfalls in ihrem Aufsatz über „Moral self-licensing and the direct touch effect" zu dem Ergebnis, dass es auch im Mutual Gift Giving Game einen ähnlichen Effekt gibt.

Joachim Weimann

Otto-von-Guericke-Universität

Magdeburg

Joachim.Weimann@ovgu.de
Die Ökonomik der Weihnachtsgeschenke lässt sich letztlich doch mit dem Fest versöhnen. Es gibt - ökonomisch gesehen - weiterhin gute Gründe, darauf zu verzichten, sich gegenseitig schön verpackte Geldscheine unter den Weihnachtsbaum zu legen. In diesem Sinne: Frohe Weihnachten. 